University of New Mexico

UNM Digital Repository

Mathematics and Statistics Faculty and Staff

Publications

Academic Department Resources

$10-2020$

True-False Set is a particular case of the Refined Neutrosophic

Set

Florentin Smarandache

Said Broumi

Follow this and additional works at: https://digitalrepository.unm.edu/math_fsp

Part of the Logic and Foundations Commons, Other Mathematics Commons, and the Set Theory Commons 


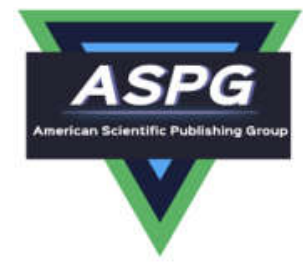

\title{
True-False Set is a particular case of the Refined Neutrosophic Set
}

\author{
${ }^{1}$ Florentin Smarandache, ${ }^{2}$ Said Broumi \\ ${ }^{1}$ Dept. Math and Sciences, University of New Mexico, Gallup, NM 87301, USA; smarand@unm.edu \\ ${ }^{2}$ Laboratory of Information Processing, Faculty of Science Ben M'Sik, \\ University Hassan II, Casablanca, Morocco; broumisaid78@gmail.com \\ Correspondence: smarand@unm.edu
}

\section{Abstract}

Borzooei, Mohseni Takallo, and Jun recently proposed a new type of set, called True-False Set [1], and they claimed it is a generalization of Neutrosophic Set [2]. We prove that this assertion is untrue. Actually it's the opposite, the True-False Set is a particular case of the Refined Neutrosophic Set..

Keywords: Refined Neutrosophic Set, True-False Set, Neutrosophic Set, Indeterminacy.

\section{Definition of True-False Set [1]}

A True-False set (TF-set), on a none-empty set $\mathrm{X}$, is a structure of the form:

$$
A_{T F S}=\left\{x ; t_{A}(x), T_{A}(x), f_{A}(x), F_{A}(x) \mid x \in X\right\} ; \text { the index "TFS" stands for True-False Set; }
$$

where $t_{A}: X \rightarrow[0,1] ; t_{A}$ represents the single-valued truth function;

$T_{A}: X \rightarrow I([0,1])$, where $I([0,1])$ is the set of all subintervals of $[0,1] ; T_{A}$ represents the interval-valued truth function;

$$
\begin{aligned}
& f_{A}: X \rightarrow[0,1] ; f_{A} \text { represents the single-valued falsehood function; } \\
& F_{A}: X \rightarrow I([0,1]) ; F_{A} \text { represents the interval-valued falsehood function. }
\end{aligned}
$$

It is not clear why two truth-functions and two falsehood-functions are needed for the same element $x$. There is no justification.

\section{Definition of neutrosophic set [2]}

We try to use similar notations and language in order to make easy comparison between the two types of sets.

Let $X$ be a non-empty universe of discourse.

A Neutrosophic Set on $X$ is a structure of the form:

$$
A_{N S}=\left\{x ; T_{A}(x), I_{A}(x), F_{A}(x) \mid x \in X\right\},
$$


where $T_{A}, I_{A}, F_{A}: X \rightarrow \mathcal{P}(]^{-} 0,1^{+}[)$, where $\mathcal{P}(]^{-} 0,1^{+}[)$is the set of all standard or nonstandard subsets of the nonstandard interval $]^{-} 0,1^{+}[$.

\section{Distinctions between True-False Set and Neutrosophic Set}

1) Clearly $\mathcal{P}(]^{-} 0,1^{+}[) \supsetneq I([0,1])$. From this point of view, the neutrosophic set is larger than the True-False Set.

$\mathcal{P}(]^{-} 0,1^{+}[)$includes not only strandard subintervals of $[0,1]$ as $I([0,1])$, but any standard subsets of $[0,1]$.

2) $\mathcal{P}(]^{-} 0,1^{+}[)$also includes non-standard subsets of $]^{-} 0,1^{+}$, left and right monads, binads from nonstandard analysis, that help make a distinction between absolute truth (truth in all possible worlds, according to Leibniz), whose truth-value is $T_{A}(x)=1^{+}$, where $1^{+}=1+\varepsilon>1$ and $\varepsilon$ is a positive infinitesimal number.

Similarly for absolute / relative indeterminacy and respectively falsehood.

The True-False Set cannot make distinctions between absolute and relative truth/falsehood.

3) Neutrosophic Set is much more complex as structure than the True-False Set; Neutrosophic Set has been further extended Neutrosophic Overset (where the neutrosophic components could be $>1$ ), Neutrosophic Underset (where the neutrosophic components could be $<0$ ), and Neutrosophic Offset (where the neutrosophic components could be $>1$ and $<0)$ in $2007 \& 2016([3]$, [4]).

\section{Transformation of a Single-Valued Neutrosophic Set to a True-False Set [1]}

The authors of [1] considered only the simplest form of the Neutrosophic Set, i.e. when the neutrosophic components T, I, F are single (crisp) numbers in [0, 1], while the general definition [2] of neutrosophic set stated since 1998 that $\mathrm{T}, \mathrm{I}, \mathrm{F}$ can be any subsets of $[0,1]$, or any nonstandard subsets of the non-standard unit interval $]^{-} 0,1^{+}[$.

They considered the single-valued neutrosophic set:

$$
A_{N S}=\left\{x ; T_{N S}(x), I_{N S}(x), F_{N S}(x) \mid x \in X\right\},
$$

where $T_{N S}, I_{N S}, F_{N S}: X \rightarrow[0,1]$ are single-valued truth, indeterminacy, and falsehood functions respectively. The index "NS" stands for Neutrosophic Set (we adjusted their Greek letter notations to Latin ones, in order to exactly match the common use notations of the neutrosophic set).

They transformed it to a True-False Set in the following way:

$$
\begin{aligned}
& t(x)=T_{N S}(x) ; \\
& f(x)=F_{N S}(x) ; \\
& T_{T F S}(x)=\left\{\begin{array}{l}
{\left[T_{N S}(x), I_{N S}(x)\right], \text { if } T_{N S}(x) \leq I_{N S}(x) ;} \\
{\left[I_{N S}(x), T_{N S}(x)\right], \text { if } I_{N S}(x) \leq T_{N S}(x) ;}
\end{array}\right. \\
& F_{T F S}(x)=\left\{\begin{array}{l}
{\left[F_{N S}(x), I_{N S}(x)\right], \text { if } F_{N S}(x) \leq I_{N S}(x) ;} \\
{\left[I_{N S}(x), F_{N S}(x)\right], \text { if } I_{N S}(x) \leq F_{N S}(x) .}
\end{array}\right.
\end{aligned}
$$

And they formed the following True-False Set:

$$
A_{T F S}=\left\{x ; t(x), T_{T F S}(x), f(x), F_{T F S}(x) \mid x \in X\right\}=\left\{\left\langle x ; T_{N S}(x), T_{T F S}(x), F_{N S}(x), F_{T F S}(x)\right\rangle \mid x \in X\right\} .
$$


This True-False Set, $A_{T F S}$, has two truth-functions and two-falsehood functions, but no indeterminacy (neutrality) function (they removed it).

Transforming the neutrosophic set $A_{N S}$ into a true-false set $A_{T F S}$ is just a mathematical artifact. It is not proven that $A_{N S}$ is equivalent to $A_{T F S}$. Actually, we'll prove below that they are not.

Other mathematical transformations can be designed as well, constructing new intervals, or combining the neutrosophic functions in other ways, etc. But the equivalence, if any, should be proven.

\section{Indeterminacy (Neutrality)}

The indeterminacy (neutrality) is the quintessence (the flavor) of neutrosophic set, that stringently distinguishes it from previous types of sets.

By eliminating the indeterminacy (or neutrality) from the neutrosophic set $A_{N S}$, when constructing a true-false set $A_{T F S}$, the true-false set $A_{T F S}$ becomes defficient, incapable of characterizing the neutrosophic triads of the form $(\langle A\rangle,\langle\operatorname{neut} A\rangle,\langle\operatorname{anti} A\rangle)$, where $\langle A\rangle$ is an item (idea, proposition, attribute, concept, etc.), $\langle$ anti $A\rangle$ is its opposite, and $\langle$ neut $A\rangle$ is the neutral between these opposites.

For example, in games we have such triads (where $\langle A\rangle=$ winning): $<$ winning, tie game, loosing $>$.

\section{Numerical Counter-Example of Transforming a Single-Valued Neutrosophic Set to a True-False Set}

Let's take only one element from a single valued neutrosophic set (for the other elements it will be similar):

$$
x_{N S}(0.3,0.4,0.2), \text { hence } T_{N S}(x)=0.3, I_{N S}(x)=0.4, F_{N S}(x)=0.2 \text {. }
$$

Let's transform it into a true-false set's element according to [1]:

$$
x_{T F S}(0.3,[0.3,0.4], 0.2,[0.2,0.4]) \text {, hence } t_{T F S}(x)=0.3, T_{T F S}(x)=[0.3,0.4], f_{T F S}(x)=0.2, F_{T F S}(x)=
$$
$[0.2,0.4]$.

The indeterminacy $I_{N S}(x)=0.4$ into the neutrosophic set has been replaced into the true-false set by an interval-value truth $T_{T F S}(x)=[0.3,0.4]$ and an interval-value falsehood $F_{T F S}(x)=[0.2,0.4]$. But these are a totally different results.

If, with respect to an element, the indeterminacy-membership is 0.4 , this is not equivalent with element's truthmembership be equal to $[0.3,0.4]$ and its false-membership be equal to [0.2, 0.4].

\section{Other Counter-Examples}

Let $x_{N S}(0.3,0.4,0.2)$ represent, with respect to the player $x$ in a game where he plays against others, that his degree of winning $\left(T_{N S}=0.3\right)$, his degree of tie game $\left(I_{N S}=0.4\right)$, and his degree of loosing $\left(F_{N S}=0.2\right)$.

By transforming $x_{N S}$ to $x_{T F S}(0.3,[0.3,0.4], 0.2,[0.2,0.4])$, we get that with respect to the same player $x$, his degree of winning is 0.3 or $[0.3,0.4]$, and his degree of loosing is 0.2 or $[0.2,0.4]$. 
7.1. Therefore, the true-false set does not provide any degree of "tie game", so this type of set is incomplete. The true-false set does not catch the middle side (neutrality, or indeterminacy) in between opposites.

7.2. Another drawback is that TFS increases the imprecision of the truth function: for $\mathrm{T}_{\mathrm{NS}}=0.3$, it gets $\mathrm{T}_{\mathrm{TFS}}$ $=0.3$ or $[0.3,0.4]$, so the truth value becomes vaguer after the TFS transformation.

TFS increases the imprecision of the falsehood function as well: for FNS $=0.2$, it gets FTFS $=0.2$ or $[0.2,0.4]$, so the falsehood value becomes vaguer after the TFS transformation.

\section{The True-False Set is a particular case of the Refined Neutrosophic Set}

In the Refined Neutrosophic Set (Logic, Probability), $\mathrm{T}$ can be split into subcomponents $\mathrm{T}_{1}, \mathrm{~T}_{2}, \ldots, \mathrm{T}_{\mathrm{p}}$, and $\mathrm{I}$ into $\mathrm{I}_{1}$, $\mathrm{I}_{2}, \ldots, \mathrm{I}_{\mathrm{r}}$, and $\mathrm{F}$ into $\mathrm{F}_{1}, \mathrm{~F}_{2}, \ldots, \mathrm{F}_{\mathrm{s}}$, where $\mathrm{p}, \mathrm{r}, \mathrm{s} \in\{0,1,2, \ldots, \infty\}$ and $\mathrm{p}+\mathrm{r}+\mathrm{s}=\mathrm{n} \in\{0,1,2, \ldots, \infty\}$. By index $=0$, of a neutrosophic component T, I, or F, or of any of their subcomponents, we denote the empty set, i.e. $\mathrm{T}_{0}=\phi, \mathrm{I}_{0}=\phi$, $\mathrm{F}_{0}=\phi$. The case $\left(\mathrm{T}_{0}, \mathrm{I}_{0}, \mathrm{~F}_{0}\right)$ is the most degenerated one. See [4].

From (T, I, F), where T, I, F are any subsets of $[0,1]$, we replace $\mathrm{I}_{0}=\phi$ (empty set), and refine/split $\mathrm{T}$ into $\mathrm{T}_{1}$ (singlevalued truth component) and $\mathrm{T}_{2}$ (as an interval-valued truth component), while $\mathrm{F}$ is similarly refined/split into $\mathrm{F}_{1}$ (as a single-valued falsehood component) and $\mathrm{F}_{2}$ (as an interval-valued falsehood component). Therefore, we replaced $\mathrm{p}$ $=2, r=0$, and $\mathrm{s}=2$ into the general form of the Refined Neutrosophic Set, and we found the True-False Set $\left(\mathrm{T}_{1}, \mathrm{~T}_{2}\right.$, $\left.\mathrm{I}_{0}=\phi, \mathrm{F}_{1}, \mathrm{~F}_{2}\right)$.

\section{Conclusion}

We proved that the transformation of the Neutrosophic Set into a True-False Set does not give equivalent results by using several coounter-examples. Also, we proved that the True-False Set is a particular case of Refined Neutrosophic Set.

\section{References}

[1] R. A Borzooei, M. Mohseni Taakallo, Y. B. Yun: True-False Sets, 2020 8th Iranian Joint Congress on Fuzzy and Intelligent Systems (FIS), September 204, 2020, Mashad, Iran, 5 pages.

[2] F. Smarandache: Neutrosophy. Neutrosophic Probability, Set, and Logic. Rehoboth: American Research Press, 1998; http://fs.unm.edu/eBook-Neutrosophics6.pdf

[3] F. Smarandache, Neutrosophic Overset, Neutrosophic Underset, and Neutrosophic Offset. Similarly for Neutrosophic Over-/Under-/Off- Logic, Probability, and Statistics, 168 p., Pons Editions, Bruxelles, Belgique, 2016; https://arxiv.org/ftp/arxiv/papers/1607/1607.00234.pdf, https://hal.archives-ouvertes.fr/hal-01340830

[4] F. Smarandache: n-Valued Refined Neutrosophic Logic and Its Applications to Physics. Progress in Physics, 143-146, Vol. 4, 2013; arXiv:1407.1041 [cs.AI] 2013; http://fs.unm.edu/RefinedNeutrosophicSet.pdf 